\title{
STUDY ON CARRIER MATERIAL OF IMMOBILIZATION \\ ACETYLCHOLINESTERASE FOR BIOSENSOR IN DETECTION OF ORGANOPHOSPHORUS PESTICIDE RESIDUES
}

\author{
Xia Sun ${ }^{1,2}$, Xiangyou Wang ${ }^{1, *}$, Chuandong Jia ${ }^{3}$ \\ ${ }^{1}$ College of Light Industry \& Agricultural Engineering, Shandong University of Technology, \\ 12 Zhangzhou Lu, Zibo, Shandong P. R. China 255049 \\ ${ }^{2}$ College of Engineering, China Agricultural University, 17 Qinghua Donglu, Beijing, P. R. \\ China. 100083 \\ ${ }^{3}$ School of Mingshui, Zhoucun Zibo Shandong, P.R. China.255318 \\ * Corresponding author: Professor Xiang-You Wang, College of Light Industry \& \\ Agricultural Engineering, Shandong University of Technology, P. O. Box 50, 12 Zhangzhou \\ Lu, Zibo 255049, Shandong P. R. China; Fax: +86-533-2786558; E-mail: \\ wxy@sdut.edu.cn.
}

Abstract: A comparison between several immobilization materials of AChE on surface of glassy carbon electrode(GCE) was presented. The immobilization methods employed crosslinking method with glutaraldehyde as a cross-linking agent and bovine serum albumin(BSA) as a protectant, AChE was immobilized on different membranes including nylon membrane, cellulose nitrate membrane and chitosan membrane respectively. The enzyme membrane was then fixed on the surface of glassy carbon electrode(GCE) with O-ring to prepare an amperometric biosensor for the detection of organophosphorus pesticides. The activity of immobilization $\mathrm{AChE}$ was detected by measuring the oxidation current of thiocholine, the results showed that the activity of immobilization $\mathrm{AChE}$ were all different with different membrane as carrier material. Compared with nylon membrane and cellulose nitrate membrane, chitosan membrane was obviously good. So chitosan membrane can be selected as immobilized AChE carrier material.

Keywords: Acetylcholinesterase, pesticides residue, biosensor, immobilization, carrier material

Please use the following format when citing this chapter:

Sun, X., Wang, X. and Jia, C., 2009, in IFIP International Federation for Information Processing, Volume 295, Computer and Computing Technologies in Agriculture II, Volume 3, eds. D. Li, Z. Chunjiang, (Boston: Springer), pp. 2283-2292. 


\section{INTRODUCTION}

Organophosphorus pesticides (OP) are very toxic compounds, they can lead to a severe impairment of nerve functions of human or even death because they inhibit main metabolic pathways (Ivanov et al., 2002, Neufeld et al., 2000). The intensive use of organophosphorus pesticides in agriculture requires further progress in the development of rapid and efficient monitoring methods. Nowadays the conventional analytical techniques mainly used for detecting organophosphorous and carbamate pesticides residues, the chromatographic ones, such as HPLC and GC,has been most commonly used as criterion methods due to their high sensitivity, reliability and precision. However these analytical techniques are time-consuming and expensive and above all, require pretreatment of the sample and highly qualified technicians (Kim et al., 2007, Schulze et al., 2002).

Biosensor techniques based on enzymatic inhibition have gained considerable attention in the past decade due to the advantages of simplicity, rapidity, reliability and low cost devices compared with the conventional analytical techniques (Andreescu et al., 2005). According to the difference of transducers, biosensors mainly included in three types: potentiometric (Marek et al. 2005), amperometric (Dan Du et al.,2007), and optical biosnsors(A.L. Simonian et al.,2005). Among these methods, amperometric biosensor is the most frequently reported because of the simple configuration, close contact between enzyme layer and transducers, high sensitivity.

Enzymes immobilization is a crucial step for the design of the biosensor. it's methods include that enzyme is immobilized on the working electrode surface directly(Arduini et al.,2006), and enzyme is immobilized on membranes, the enzyme membrane is then fixed on the surface of electrode (Fuxiang Wei et al.,2007). Appropriate selection of the electrode, membranes material and immobilization methods is essential for a reliable biosensor, because it will strongly affects the performance of the biosensor in term of sensitivity, stability, response time and reproducibility.

In this work, a comparison of the AchE activity after immobilization with different materials in biosensors was presented. The immobilization methods employed crosslinking method with glutaraldehyde, and under the immobilization methods, the carrier materials were explored, AChE was immobilized on different membranes that were nylon membrane, cellulose nitrate membrane and chitosan membrane respectively. The enzyme membrane was then fixed on the surface of GCE to prepare an amperometric biosensor. The results were evaluated by electrochemical measurements, the results showed that the rate recovery activity of enzyme immobilized with different membrane was different, the activity of enzyme immobilized with chitosan membrane was the highest, and the activity of enzyme immobilized with nylon membrane was the worst. 


\section{MATERIALS AND METHODS}

\subsection{Apparatus}

Specrophotometric measurements were performed on UV-Vis spectrophotometer purchased from DaoJin Japan, high-speed freezing centrifuge was supplied from $\operatorname{Sigma}(3 \mathrm{~K} 15$, Germany); Acidometer was the product of Vastell $(230 \mathrm{~A}+, \mathrm{USA})$; JJ-2 tissue triturator from Ronghua instrument manufacture Co. (Jiangsu,China), DK-98- I galvanothermy constant temperature boiler was obtained from Taisite instrument Co. (Tianjin,China). Electrochemical measurements were performed on an CHI600 Electrochemical Workstation from Shanghai Chenhua instrument Ltd.(Shanghai,China). 3- electrode was purchased from Aida Hengsheng technology Co.(Tianjin, China). glassy-carbon electrode with $3 \mathrm{~mm}$ diameter as working electrode, platinum electrode as counter electrode, saturated calomelelectrode(SCE)as reference electrode.

\subsection{Chemicals and reagents}

Chicken brain-esterase was extracted from living chicken bought from market. Acetylthiocholine chloride (ATCh) as substrates was purchased from Nuoyawei Biology Tech.Co.(Shanghai,China). 5,5'-dithiobis(2'nitrobenzoic acid) (DTNB) as color react of reagent provided by Sigma. Glutaraldehyde and BSA were provided by Sigma. Dichlorvos( $40 \%$ milk grease)was the product of Dacheng pesticide(Shandong,China). All other chemicals were of analytical grade and were used without further purification. Distilled water was used throughout for the preparation of solutions.

\subsection{Preparation and extraction of the chicken brain esterase}

Chicken brain-esterase was extracted using a method, which was from a previously reported technique (Sunxia et al.,2008). The fresh chicken brain with phosphate buffer ( $1 \%$ Triton X-100,pH8.0, $0.1 \mathrm{~mol} / 1)$ in the ratio of $1 \mathrm{~g} / 3$ $\mathrm{ml}$ is homogenized for $40 \mathrm{~min}$ at $8000 \mathrm{rpm}$ at $4^{\circ} \mathrm{C}$ with a tissue triturator. And the chicken brain-esterase solution is obtained from the final supernatant after centrifugation and then preserved in refrigerator at $4^{\circ} \mathrm{C}$.

For measuring AChE activity was modified from the assay described by Ellman (Ellman et al.,1961). The Ellman method has found widespread 
application for determination of cholinesterase (ChE) activities using acetylthiocholine (ACTC) as substrates (T. Hamers et al.,2000). Ellman method is based on the increase of yellow colour nitrobenzoate (TNB) ion produced from thiocholine when it reacts with dithiobisnitrobenzoate (DTNB) ion (Eq. 1 and 2, below) (Zou et al.,2006).

$$
\begin{aligned}
& \text { acetylcholine }+\mathrm{H}_{2} \mathrm{O} \underset{\text { Thiocholine }+ \text { acetic acid }}{\longrightarrow} \stackrel{\mathrm{AChE}}{\longrightarrow} \text { Formation of TNB } \\
& \text { Tyellow colour }(412 \mathrm{~nm})]
\end{aligned}
$$

The calculation of enzyme activity of formation is described as followed:

$$
\mathrm{E}\left(\mu \mathrm{mol} \cdot \mathrm{min}^{-1} \cdot \mathrm{mL}\right)=V \times A \times 10^{6} /(v \times K \times L)
$$

where $\mathrm{E}$ is the activity of enzyme, $\mathrm{V}$ is the volume of enzyme liquid $(\mathrm{mL})$, $\mathrm{v}=0.02 . \mathrm{K}$ is extinction coefficient $(\mathrm{L} / \mathrm{mmol} \cdot \mathrm{mm}), K=1.36 . L$ is light length of enzyme liquid (10mm). $v$ is total volume of reaction of liquid $(\mathrm{mL})$, $v=3.14$. Every experiment was done replicatively in triplicate. After calculated the activity of chicken brain-esterase, recorded and stocked at $4^{\circ} \mathrm{C}$ for use.

\subsection{Preparation of immobilization enzyme membrane}

\subsubsection{Pretreatment of carrier material}

Carrier material was pretreated using a method, which was slightly modified from a previously reported technique (Fuxiang Wei et al.,2007). Commercial carrier material were made into circular sheet about $10 \mathrm{~mm}$ diameter, and employed the following method of activation respectively. nylon carrier was immersed in anhydrous methanol for $30 \mathrm{~min}$, then washed 5 min with phosphate buffer $(0.1 \mathrm{~mol} / 1, \mathrm{pH} 8.0)$, naturally dried and stock for use. Cellulose acetate porous membrane and cellulose nitrate porous membrane were immersed in phosphate buffer $(0.01 \mathrm{~mol} / 1, \mathrm{pH} 7.0)$ for $48 \mathrm{~h}$ at $4^{\circ} \mathrm{C}$.

\subsubsection{Preparation of chitosan porous membrane}

The preparation method of chitosan porous membrane was modified from the assay described by Zhibin Qiang (Zhibin Qiang et al.,2007).Weighing $0.1 \mathrm{~g}$ chitosan powder,then added $10 \mathrm{ml}$ of acetate solution $(1 \%, \mathrm{~m} / \mathrm{m})$, and 
mixed round for $10 \mathrm{~min}$ to yellow sol.Then centrifugal for $5 \mathrm{~min}$ in highspeed freezing centrifuge at $3000 \mathrm{r} / \mathrm{min}$ at room temperature to remove insoluble particles. Then the pretreated cellulose nitrate porous membrane was immersed in the sol for $12 \mathrm{~h}$, and then immersed in phosphate buffer $(0.1 \mathrm{~mol} / \mathrm{L}, \mathrm{pH} 8.0)$ for 12 , dried and stock for use.

\subsubsection{Immobilization of enzyme}

Chicken brain-AChEs, diluted at a concentration of $100 \mathrm{U} \mathrm{mL}^{-1}$ in phosphate buffer $(0.1 \mathrm{~mol} / \mathrm{L}, \mathrm{pH} 8.0)$, were immobilized on sensitive membrane. The immobilization method of enzyme was modified from the assay described by Fuxiang Wei (Fuxiang Wei et al.,2007), the protocol were performed according to the following procedure: $10 \mathrm{U}$ of AchE liquid and $30.0 \mu \mathrm{L}$ of $\mathrm{BSA}(1.0 \%), \quad 2.0 \mu \mathrm{L}$ of glutaraldehyde $(5.0 \%)$ in $0.50 \mathrm{~mL}$ of centrifuge tube, were blended in vortex mixer. Then nylon membrane, cellulose nitrate membrane and chitosan membrane were immersed in 0.50 $\mathrm{mL}$ of centrifuge tube which contained those enzyme liquid for $8 \mathrm{~h}$ at $4^{\circ} \mathrm{C}$ respectively. And enzyme membranes was washed with phosphate buffer $(0.1 \mathrm{~mol} / \mathrm{L}, \mathrm{pH} 8.0)$, and then dried at room temperature, and determined enzyme activity by electrochemical measurements.

\subsection{Amperometric measurement of AChE enzyme activity}

\subsubsection{Amperometric measurement principle}

The detecting principle is that pesticides can be measured by comparing the residual activity of the enzyme after exposure to OP compounds with the initial activity. After exposure to OP compounds, enzyme activity is decreased, depending on the concentration and the time of exposure. The enzyme activity is estimated amperometrically at about between $100 \mathrm{mV}$ and $700 \mathrm{mV}$ versus reference electrode by the anodic oxidation of the thiocholine (TCh) produced by hydrolysis of the acetylthiocholine (ATCh) substrate. (Eq. 3 and 4, below) (Lihong Wang et al.,2006.)

$$
\text { acetylcholine }+\mathrm{H}_{2} \mathrm{O} \stackrel{\mathrm{AChE}}{\longrightarrow} \text { thiocholine }+ \text { acetic acid }
$$


The following general expression of enzyme-substrate-inhibitor is used to obtain a quantitative estimate of the relation between dichlorvos concentration and the degree of inhibition. The remaining activity (I\%) is determined according to the following formula: $\mathrm{I} \%=((\mathrm{I} 0-\mathrm{I} 1) / \mathrm{I} 0) \times 100 \%$

Where $I \%$ is the degree of inhibition related to the inhibitor concentration. I0 is the initial current (I0) of the biosensor is measured without inhibitor in phosphate buffer. I1 is the current after the incubation in the phosphate buffer with different concentrations of inhibitor(Dan Du et al., 2007).

\subsubsection{Amperometric measurement of free AChE enzyme activity}

Firstly, the surface of GCE was polished by $0.05 \mu \mathrm{m}$ alumina suspension to a mirror finish. Secondly, 3-electrode connected with electrochemical workstation was immersed in $10 \mathrm{ml}$ of electrochemical reaction cell in which $9 \mathrm{ml}$ of phosphate buffer $(0.1 \mathrm{~mol} / \mathrm{L}, \mathrm{pH} 7.5)$ as electrolyte was added, and then different concentration $\mathrm{AChE}$ liquid was added respectively. Then electrochemical workstation was run, the electrodes were tested with electrical current-time at a working potential of $+600 \mathrm{mV}$ versus SCE. After the current was stable, $0.5 \mathrm{ml}$ of ATCh was injected, the change of current produced by the oxidation of thiocholine was observed. The cell was washed with distilled water between measurements.

\subsubsection{Amperometric measurement of immobilization AChE enzyme activity}

The procedure amperometric measurement of immobilization AChE enzyme activity was similar to the amperometric measurement of free $\mathrm{AChE}$ enzyme activity basically, only one was difference, substituting AChE liquid for the enzyme membrane fixed on the surface of glassy carbon working electrode with O-ring.

\section{RESULTS}

\subsection{The current of catalytic reaction under free $\mathrm{AChE}$}

The current which corresponds to the oxidation of thiocholine, the product of the enzymatic hydrolysis of ATCh substrate under different concentration AChE liquid was recorded after plateau. The relation curve between AChE concentration and electrical current volume was drawn. The curve shown in Fig1 indicated that the relation between the current value and enzyme activity was obvious linear correlation. And its linear correlation coefficient 
was 0.9945 . So the current value of catalytic reaction can be used to measure enzyme activity.

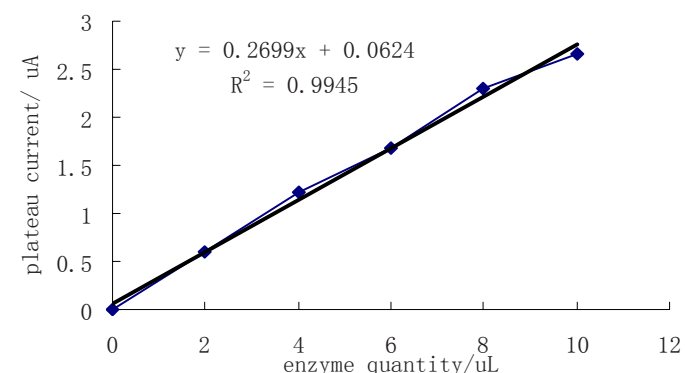

Fig. 1 The relation curve between AchE concentration and electrical current volume

\subsection{The current of catalytic reaction under immobilization AchE employed different carrier materials}

The enzyme membrane fixed on the surface of glassy carbon working electrode with O-ring. 3-electrode connected with electrochemical workstation was immersed in $10 \mathrm{ml}$ of electrochemical reaction cell in which $9 \mathrm{ml}$ of phosphate buffer $(0.1 \mathrm{~mol} / \mathrm{L}, \mathrm{pH} 7.5)$ as electrolyte was added. Then electrochemical workstation was run, the electrodes were tested with electrical current -time at a working potential of $+600 \mathrm{mV}$ versus saturated calomelelectrode. After about 50 second, electrical current reached a plateau, $0.5 \mathrm{ml}$ of ATCh was injected at this time.

\subsubsection{The current of catalytic reaction employed nylon porous membrane}

The curves shown in Fig. 2 indicated that $0.5 \mathrm{ml}$ of ATCh was injected at 50 second, and at 120 second the current began descend. At 300 second, the current achieved to valley value that was $1.643 \mathrm{uA}$.

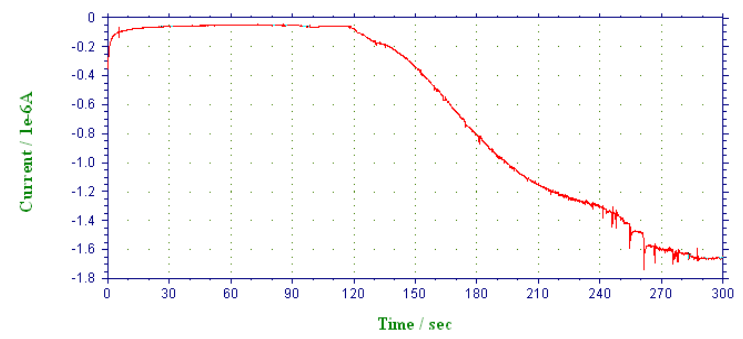

Fig.2 The current curve of catalytic reaction employed nylon membrane 


\subsubsection{The current of catalytic reaction employed cellulose nitrate porous membrane}

The curves shown in Fig. 3 indicated that $0.5 \mathrm{ml}$ of ATCh was injected at 50 second, and at 60 second the current began descend. At 240 second, the current achieved to valley value that was $1.12 \mathrm{uA}$.

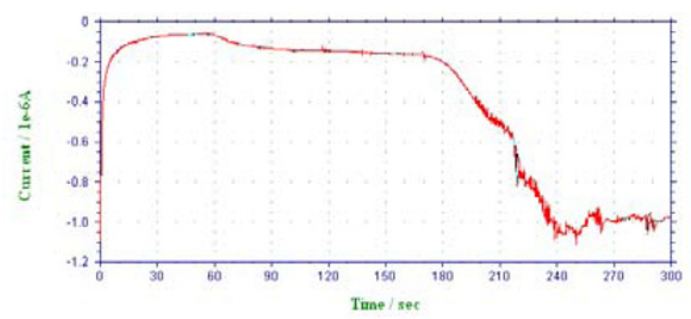

Fig. 3 The current curve of catalytic reaction employed cellulose nitrate membrane

\subsubsection{The current of catalytic reaction employed chitosan porous membrane}

The response curve for the current of catalytic reaction employed chitosan membrane was shown in Figure4. The curves showed that at 50 second, $0.5 \mathrm{ml}$ of ATCh was injected, and at 120 second the current began descend. At 180 second, the current achieved to valley value that was $2.4 \mathrm{uA}$.

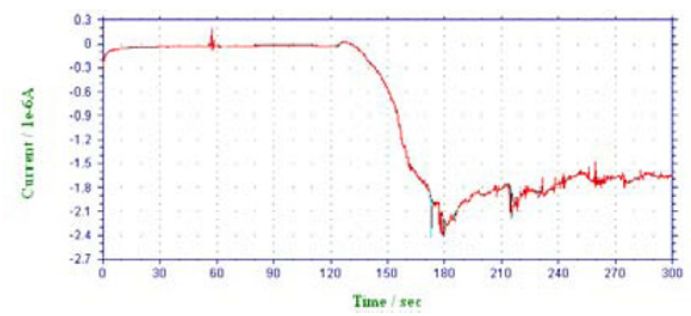

Fig. 4 The current curve of catalytic reaction employed chitosan porous membrane

\subsection{Discussion}

The results showed that the current of response time, stability time and the current stability values were all different in fig.2, fig.3, fig.4 respectively. In other words, the selection of carrier materials directly effect the activity, stabilty of immobilization enzyme. Compared with nylon membrane, the time of the current achieved to valley value immobilized with cellulose nitrate membrane was different, it showed that substrate diffusion speed in cellulose nitrate membrane was quicker than nylon membrane. But the valley value in fig2 was bigger than fig3, it showed that cellulose nitrate 
membrane osmosis was better than nylon membrane, so cellulose nitrate membrane absorption enzmye quantity less than nylon membrane, after the cellulose nitrate membrane modified by chitosan, the situation improved greatly in fig4. The reason is that there is amino structure in chitosan molecular structure, that have high affinity, that can easy to fix the enzmye.

\section{CONCLUSION}

The results were evaluated by catalytic reaction showed that the rate recovery activity of enzyme employed different membrane as carrier material was different, chitosan membrane was the highest compared with cellulose nitrate membrane and nylon membrane. And the results also showed that chitosan membrane prevented leakage of the enzmye, improved the activity of immobilization enzmye. So the AChE immobilized with chitosan membrane used in biosensor can improve the performance of the biosensor in term of sensitivity, stability, response time and reproducibility. we also can conclude that promote the application of immobilized enzymes in pesticide residue rapid detecting fields by improving traditional materials, design and exploit new carrier materials. In the future, the design and preparation of carrier materials with excellent integration performances will be one of the most significant researches for the development of immobilized enzymes.

\section{ACKNOWLEDGEMENTS}

The authors are gratefully acknowledging the financial support of the Science and Technology Research Key Program of Shandong province(No.2008GG10009027), and the Technology Program of Shandong Provincial Education Department(No. J07YF11).

\section{REFERENCES}

A.L. Simonian, T.A. Good, S.-S. Wang, \& J.R. WildNanoparticle-based optical biosensors for the direct detection of organophosphate chemical warfare agents and pesticides. Analytica Chimica Acta, 2005, 534:69-77.

Andreescu, D., Andreescu, S., Sadik, O. A., \& Gorton, L. Chapter 7 New materials for biosensors, biochips and molecular bioelectronics. In Comprehensive Analytical Chemistry, 2005, 44: 285-327. 
Avramescu, A., Andreescu, S., Noguer, T., Bala, C., Andreescu, D., \& Marty, J.-L. Biosensors designed for environmental and food quality control based on screen-printed graphite electrodes with different configurations. Analytical and Bioanalytical Chemistry, 2002,374(1):25-32.

Dan Du, Xi Huang, Jie Cai, \& Aidong Zhang. Amperometric detection of triazophos pesticide using acetylcholinesterase biosensor based on multiwall carbon nanotube-chitosan matrix Sensors and Actuators B: Chemical, 2007, 127( 2): 531-535.

Ellman, G. L., Courtney, K. D., Andres, V., \& Featherstone, R. M. A new and rapid colorimetric determination of acetylcholinesterase activity. Biochemical Pharmacology, 1961,7(2):88-90.

Fuxiang Wei, Zhenchuan Wang, \& Jinmei Wang, Determination of Organophosphorus Pesticide Residues by AnAcetylcholinesterase Biosensor in Vegetables and Fruits, Food science, 2007,28(2):229-231.

Ivanov, A. N., Lukachova, L. V., Evtugyn, G. A., Karyakina, E. E., Kiseleva, S. G., Budnikov, H. C., et al. Polyaniline-modified cholinesterase sensor for pesticide determination. Bioelectrochemistry, 2002,55(1-2):75-77.

Kim, N., Park, I.-S., \& Kim, D.-K. High-sensitivity detection for model organophosphorus and carbamate pesticide with quartz crystal microbalance-precipitation sensor. Biosensors and Bioelectronics, 2007, 22(8):1593-1599.

Lihong Wang, Lin Zhang, \& Huanlin Chen Enzymatic Biosensors for Detection of Organophosphorus Pesticides. Progress in chemistry, 2006,18(4): 440-451.

Marek Trojanowicz, Michael L. Hitchman. Determination of pesticides using electrochemical biosensors. TrAC Trends in Analytical Chemistry, 1996,15 (1):38-45.

Neufeld, T., Eshkenazi, I., Cohen, E., \& Rishpon, J. A micro flow injection electrochemical biosensor for organophosphorus pesticides. Biosensors and Bioelectronics, 2000, 15(56):323-329.

Schulze, H., Schmid, R., \& Bachmann, T. Rapid detection of neurotoxic insecticides in food using disposable acetylcholinesterase-biosensors and simple solvent extraction. Analytical and Bioanalytical Chemistry, 2002,372(2):268-272.

Siriwan Suwansa-ard, Proespichaya Kanatharana, Punnee Asawatreratanakul, Chusak Limsakul, Booncharoen Wongkittisuksa, \& Panote Thavarungkul. Semi disposable reactor biosensors for detecting carbamate pesticides in water.Biosensors and Bioelectronics, 2005, $21,(3): 445-454$.

Sunxia, Xiangyou Wang, Xiao yu Wang, \& Zhe Liu. A Comparative Study of Sensitivity of Acetylcholinesterase in Detection of Organophosphorus Pesticide Residues. International Journal of Food Engineering, 2008,4(3), article 7.

T. Hamers, K.R.J. Molin, J.H. Koeman and A.J. Murk, A small-volume bioassay for quantification of the esterase inhibiting potency of mixtures of organophosphate and carbamate insecticide in rainwater: development and optimization, Toxicological Science 2000,58:60-67.

Vangelis G. Andreou, Yannis D. Clonis A portable fiber-optic pesticide biosensor based on immobilized cholinesterase and sol-gel entrapped bromcresol purple for in-field use. Biosensors \& Bioelectronics, 2002,17:61-69.

Zhibin Qiang, Yinsheng Chen, Huawen Guo, \& Jianqi Liu, Property of Different Molecular Weight and Degree of Deacetylation Chitosan Membranes, Journal of Dong Hua University, 2007, 33(2):212-215.

Zou, M.-Q., Yang, R., Wang, D.-N., Li, J.-F., \& Jin, Q.-H. A novel immobilized cholinesterase for on-site screening of organophosphate and carbamate compounds. Pesticide Biochemistry and Physiology, 2006,86(3): 162-166. 